REVISTA CIENCIAS BIOMÉDICAS

PRESENTACIÓN DE CASOS CLÍNICOS

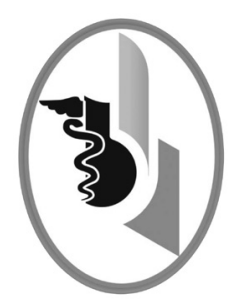

\title{
XANTOGRANULOMA JUVENIL: REPORTE DE CASO
}

\author{
JUVENILE XANTHOGRANULOMA: \\ CASE REPORT
}

\author{
Buendía-De-Ávila María $\mathrm{E}^{1}$ \\ Álvarez-Pereira Erick ${ }^{2}$ \\ Bertel-Rodríguez Daniela ${ }^{3}$ \\ Redondo-De Oro Katherine ${ }^{4}$ \\ Correspondencia: maryelenab@gmail.com \\ Recibido para evaluación: marzo-20-2015. Aceptado para publicación: octubre-25- 2016
}

\section{RESUMEN}

Introducción: el xantogranuloma juvenil (XGJ) se encuentra dentro de las histiocitosis de células de Langerhans. Es descrita como una enfermedad benigna, caracterizada por la presencia de neoformaciones pápulo-nodulares en la primera infancia (rara en los adultos) y tiene un carácter autolimitado y autoinvolutivo. Raramente afecta órganos internos; histológicamente se caracteriza por inclusiones lipídicas en histiocitos sin alteraciones metabólicas de base. El objetivo es presentar el caso de una preescolar femenina que consultó el servicio de Dermatología Pediátrica por lesión en región axilar. Caso clínico: paciente femenina de dos años y diez meses de edad con antecedentes de nacimiento a término sin complicaciones y esquema de vacunación completo para su edad. Requirió hospitalización a los cinco meses de edad por bronquiolitis aguda durante cinco días, sin necesidad de ingresar a unidad de cuidados intensivos, ni ventilación mecánica. La paciente presentaba antecedente paterno de rinitis alérgica.

Conclusión: las lesiones dermatológicas sugestivas de XG] deben tratarse si causan síntomas o para establecer su diagnóstico. Usualmente son benignas, aunque existen condiciones especiales a considerar. Rev.cienc.biomed. 2016;7(2):306-309.

\section{PALABRAS CLAVE}

Xantogranuloma Juvenil; Histiocitosis de Células no Langerhans; Dermatología.

1 Médico. Estudiante de Postgrado. Pediatría. Facultad de Medicina. Universidad de Cartagena. Colombia.

2 Médico. Especialista en Dermatología. Docente. Departamento de Pediatría. Facultad de Medicina. Universidad de Cartagena. Colombia.

3 Médico. Estudiante de Postgrado. Patología. Facultad de Medicina. Universidad de Cartagena. Colombia.

4 Médico. Especialista en Patología. Docente. Facultad de Medicina. Universidad de Cartagena. Colombia. 


\section{SUMMARY}

Introduction: juvenile xanthogranuloma (JXG) is found within histiocytosis of Langerhans cells. It is described as a benign illness characterized by the presence of nodular papules neoformations in the early childhood (rarely in adults). It has a self-limited characteristics. Rarely, this illness affects internal organs; histologically is characterized by lipid inclusions in histiocytes without basic metabolic alterations. The case is about a pre-school female child, who consulted the Pediatric Dermatology service due to axillary injury.

Case report: two years-old female patient with medical history of term birth without complications and completed immunization schedule, according to her age, required hospitalization at 5 months of age for acute bronchiolitis during 5 days, without the need to enter the Intensive Care Unit or mechanical ventilation. The patient presented paternal medical history of allergic rhinitis.

Conclusion: dermatological lesions suggestive of JXG should be treated if they cause symptoms or to establish a diagnosis. Usually they are benign, although there are special conditions to consider. Rev.cienc.biomed.2016;7(2):306-309.

\section{KEYWORDS}

Xanthogranuloma Juvenile; Histiocytosis Non-Langerhans-Cell; Dermatology.

\section{INTRODUCCIÓN}

El xantogranuloma juvenil (XGJ) fue descrito inicialmente por Adamstown en 1905, bajo el término de xantomas múltiples congénitos, término cambiado luego a nevo xantoendotelioma por Mac Donagh en 1912. Con respecto a su etiología, en 1919, Helwig y Hackney desmintieron la relación de esta patología con nevos o células endoteliales. Luego, en 1954, cuando demostraron su origen fibrohistiocítico, fue denominado xantogranuloma juvenil (1).

Es una rara enfermedad que comprende la más frecuente de las histiocitosis no Langerhans (o tipo II). El $70 \%$ de las lesiones aparecen en el primer año de vida, incluyendo el 34\% que se encuentra desde el momento del nacimiento, mientras que solo un $15 \%$ o menos, se dan en la adolescencia o en la vida adulta $(1,2)$.

\section{CASO CLÍNICO}

Paciente femenina, de dos años y diez meses de edad con antecedentes de nacimiento a término sin complicaciones y esquema de vacunación completo para su edad, requirió hospitalización a los cinco meses de edad por bronquiolitis aguda durante cinco días, sin necesidad de ingresar a unidad de cuidados intensivos. Adicionalmente, la paciente presentaba antecedente paterno de rinitis alérgica y también cuadros respiratorios bronco-obstructivos a repetición, sin requerimientos de ingreso a urgencias, ni hospitalización.

Durante la consulta se encontró dermatosis localizada en axila izquierda, lesión única constituida por una neoformación de $0.4 \mathrm{~cm}$ de diámetro, cupuliforme, de color parduzco, dolorosa con dos meses de evolución. En el examen físico la consistencia era cauchosa y sin sangrado a la manipulación. Figura No 1.

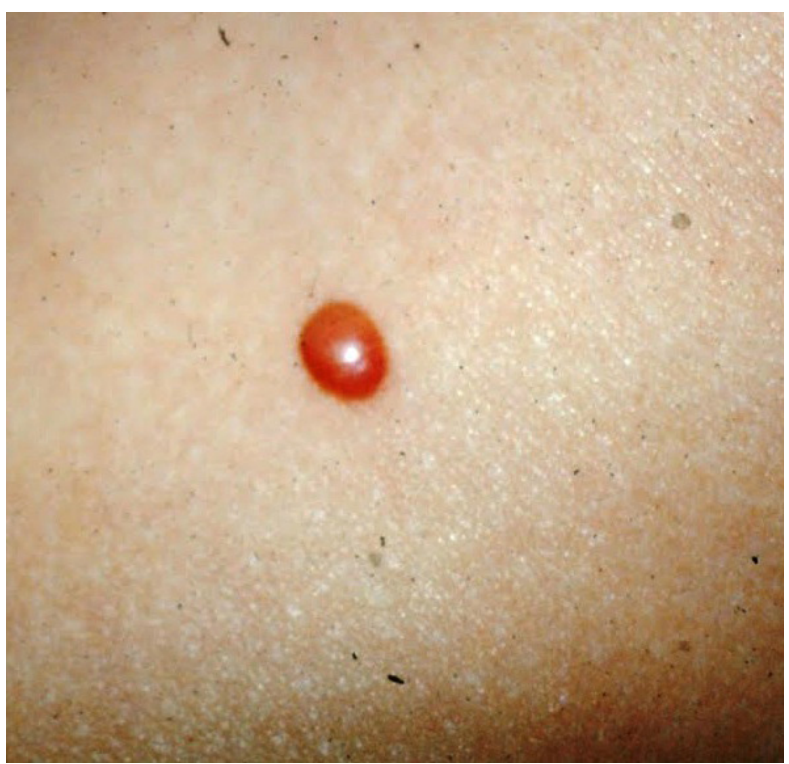

Figura No1. Lesión en región axilar de $0.4 \mathrm{~cm}$ de diámetro, consistencia cauchosa, color parduzco y sin sangrado. 

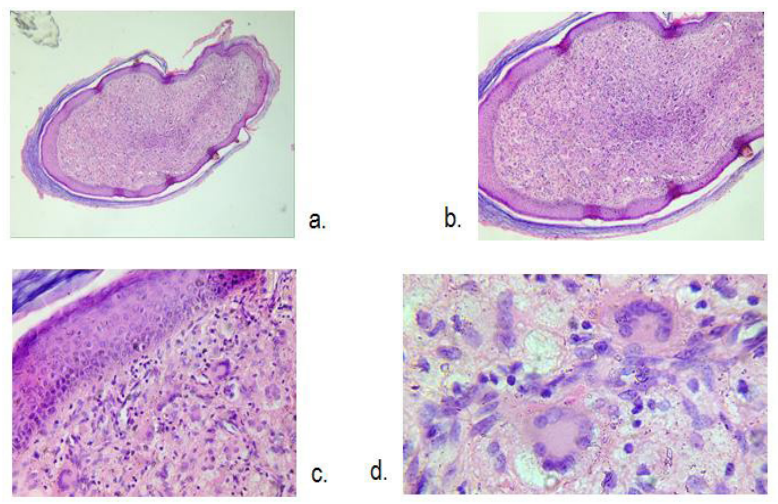

Figura N02. Histología de la lesión. Infiltrado por células linfocitarias, mezcladas con histiocitos espumosos y algunas células de Touton. No se observaron atipias. Xantogranuloma juvenil.

Al momento de la consulta, la madre de la paciente refería que la lesión había aparecido de un tamaño menor y había aumentado progresivamente, produciendo dolor. No se observaron alteraciones en otros lugares, ni compromisos oculares. Se realizó impresión diagnóstica de tumor benigno en tronco. Para establecer el diagnóstico, se decidió realizar biopsia excisional bajo anestesia general. El procedimiento se cumplió un mes después de la consulta y la lesión fue enviada a patología. No se presentaron complicaciones intra ni postoperatorias.

En la valoración histológica se observaba denso infiltrado histiocítico, algunos xantomisados y otros de aspecto estrellado, asociados a la presencia de células gigantes multinucleadas de tipo cuerpo extraño, Touton y Langhans. No se observó malignidad en la muestra. Estos hallazgos definieron el diagnóstico de XGJ. Figura $\mathrm{N}^{\circ} 2$.

Al siguiente mes la paciente fue valorada nuevamente en la consulta, con mejoría total de los síntomas. Se explicó a la madre el carácter benigno del tumor. Se dio de alta por no requerir otra intervención por este servicio.

\section{DISCUSIÓN}

El XGJ es un tipo de lesión única en un 60$80 \%$ de los casos y afecta principalmente la cabeza y el cuello, pero con la posibilidad de comprometer cualquier región corporal. Cuando se presenta afección única se conoce como XGJ solitario. Se ha descrito que afecta más a los lactantes varones (1.5 veces) que a niñas, pero al llegar a la edad adulta no hay distinciones por sexo $(3,4)$.

Son pápulas o lesiones tuberosas, ovales, bien delimitadas, de consistencia firme, de color rojo a naranja, pero que pueden ser parduzcas o eritematosas. Pueden desarrollar telangiectasias en la superficie. Por otro lado, entre los síntomas que el paciente puede manifestar están prurito intenso, dolor y, aunque es infrecuente, ulceración y sangrado $(1,3)$.

El XGJ tiene dos presentaciones: micronodular con múltiples lesiones de 1-10 mm de diámetro y la macronodular, con menor cantidad de lesiones y mayor tamaño pudiendo alcanzar hasta dos $\mathrm{cm}$ de diámetro. Aunque la primera es la forma más frecuente, hasta en un $60 \%$, es posible que coexistan las dos presentaciones en un mismo individuo (5-7).

Raramente afectan mucosas, pero pueden aparecer en menores de tres años. Cuando se localizan en tejido celular subcutáneo pueden observarse como masas blandas, bien delimitadas, no dolorosas $(1,6)$.

El compromiso extracutáneo, aunque raro, está bien documentado. Descrito principalmente como compromiso ocular con lesión uveal. Predominan en menores de dos años y tienen la capacidad de afectar iris, cuerpo ciliar, epiesclera y órbita. La complicación más temida en estos casos es la hemorragia de cámara anterior, con glaucoma secundario. Con frecuencia, el pulmón y el hígado son los órganos más afectados con lesiones viscerales asintomáticas, pero con alto riesgo de complicaciones (5).

El XGJ se ha visto asociado a otro tipo de patologías, tal es el caso de la neurofibromatosis tipo I, o sea, coexistencia de manchas "café con leche" con las lesiones del XGJ. También se ha relacionado con la leucemia mielocítica crónica juvenil, con presencia de múltiples lesiones y en algunos casos de forma solitaria. Se ha descrito que el origen de estas asociaciones se encuentra en el gen que estimula la colonia de granulocitos en el cromosoma 17q (1). 
ISSN: 2215-7840, 7(2), julio-diciembre 2016, Buendía-De-Ávila María E, Álvarez-Pereira Erick, Bertel-Rodríguez Daniela, Redondo-De Oro Katherine

Histopatológicamente se caracteriza el XGJ por denso infiltrado dérmico de histiocitos, que pueden encontrarse vacuolados, xantomizados con citoplasma espumoso, fusiformes con patrón estoriforme, festoneados con aspecto estrellado y oncocíticos con citoplasma eosinófilo finamente granular. En las formas cutáneas se pueden encontrar células gigantes multinucleadas que pueden ser: [A] Tipo cuerpo extraño. [B] Tipo Touton (tienen citoplasma homogéneo central con una corona de núcleos con citoplasma excéntrico xantomizado, es el hallazgo histopatológico más específico de las lesiones cutáneas. [C] Tipo vidrio esmerilado con citoplasma eosinófilo transparente. Estas células se distribuyen en infiltrados nodulares dérmicos mal delimitados, que pueden extenderse a tejido celular subcutáneo, incluso músculo (9-10).

Con marcadores inmunohistoquímicos se observa que los histiocitos expresan CD68 en el $100 \%$ de los casos, vimentina, factor XIIIa, y lisozima; mientras son negativos para CD34, actina muscular lisa, S-100 (aunque hasta un $10 \%$ pueden expresarlo) y CD1a.

El principal diagnóstico diferencial de esta lesión debe hacerse con los xantomas, especialmente, cuando aparecen múltiples lesiones. Se debe diferenciar de otras histiocitosis. Cuando en el patrón histológico se observe alguna atipia, siempre se debe descartar leucemia, linfoma o sarcoma de células redondas (8).

El pronóstico de XGJ es favorable al tratarse de un proceso benigno, se puede revertir entre 3-6 años. Esta clase de tumores tienen bajo índice de recidivas que no alcanza el $7 \%$. Tras resecciones incompletas se han descrito involuciones completas (6-8). El tratamiento va dirigido a la extirpación de la lesión sea por fines estéticos o para confirmar el diagnóstico. Se recomienda tratar siempre las lesiones oculares para evitar complicaciones secundarias (1).

\section{CONCLUSIÓN}

XGJ es una patología dermatológica que se debe diagnosticar para confirmar que posee condiciones de benignidad. Se deben identificar aquellas situaciones que pueden generar aspectos de riesgo, las asociaciones con otras patologías y las afecciones oculares.

CONFLICTO DE INTERESES: ninguno que declarar.

FINANCIACIÓN: recursos propios de los autores. Estudios de laboratorio, insumos hospitalarios, medicamentos y honorarios profesionales aportados dentro de la atención asistencial.

\section{REFERENCIAS BIBLIOGRÁFICAS}

1. Rodríguez-Peralto JL, Alonso S, Carrillo R. Tema 155: Xantogranuloma juvenil. Dermatología: Correlación clínico-patológica. [Acceso 23 de octubre del 2015]. Disponible en: http:// studylib.es/doc/6160562/tema-155-xantogranuloma-juvenil

2. Martínez-Estrada V, García Salazar V, Navarrete-Franco G. Xantogranuloma juvenil. Reporte de un caso. Rev Cent Dermatol Pascua 2002;11(1):22-26.

3. Buján MM, Sosa G, Bettina-Cervini A, Laterza A, Pierini AM. Xantogranuloma juvenil: experiencia en un hospital pediátrico. Dermatología Argentina. 2012:4(16):262-67.

4. Collins L, Banks R, Robinson M. Juvenil xanthogranuloma: unusual intraoral finding. British J Oral and Maxillofacial Surgery. 2015;53:86-8.

5. Tamir I, Davi R et al. Solitary juvenile xanthogranuloma mimicking intracranial tumor in children. Case Reports. J Clinical Neuroscience. 2013;20:183-188.

6. Hagmann C, El-Bahrawy M, Stamp G, Abel RM. Juvenile xanthogranuloma: a case report of a preterm baby. J Pediatric Surgery. 2006;41:573-75.

7. Yahya S, Jouini R, Maazoun $\mathrm{K}$, Krichéne I, et al. Images en médecine: xanthogranulome juvénile. Presse Med. 2008;37:1180-1181.

8. Simon-Cypel TK, Marvin-Zuker R. Juvenil xantogranuloma: case report and review of the literature. Can J Plast Surg. 2008;16(3):175-77.

9. Patel B. Juvenile xanthogranuloma. [Acceso 23 de octubre del 2015]. Disponible en: http:// emedicine.medscape.com/article/1209681-overview

10. Monahan S. Dermatologic manifestations of juvenil xanthogranuloma. [Acceso 23 de octubre del 2015]. Disponible en: http://emedicine.medscape.com/article/1111629-overview 